\title{
A Theoretical Analysis of Vibration Power Absorption Density in Human Fingertip
}

\author{
J.Z. Wu, R.G. Dong, D.E. Welcome, X.S. Xu \\ Health Effects Laboratory, National Institute for Occupational Safety and Health \\ Morgantown, West Virginia, USA
}

\section{Introduction}

The effect of vibration exposure is generally frequency-dependent. However, a reasonable frequency weighting that accounts for any component of the vibration-induced finger disorders, such as the most studied vibration-induced white finger, has not been well established [1]. The current frequency weighting was formulated based on the subjective sensation of the entire handarm system measured under some specific testing conditions [2]. We hypothesize that vibration power absorption density (VPAD) is a good measure of the finger vibration exposure, and it may be used to help develop a better frequency weighting for assessing the risk of the finger disorders. We propose a hybrid modeling method for analyzing the VPAD of the finger soft tissues.

\section{Method}

The hand-finger system is simulated by using a hybrid model, which combines a lumped parameter model with a two-dimensional finite element (2D FE) model, as illustrated in Fig. 1. The fingertip is simulated using a 2D FE model, while the effective mass of the handfinger is represented by the mass element $m$. The coupling between the fingertip and hand-finger is represented by the spring and damping element $(k 1$ and $c 1)$. The contact between the fingertip and the vibrating plate is simulated in the FE modeling, while the coupling between the hand and the vibrating plate is represented by another spring/damping unit ( $k 3$ and $c 3$ ). The coupling between hand, forearm, and ground is represented using a spring/damping unit ( $k 2$ and $c 2$ ). The fingertip model was assumed to be composed of skin layers, subcutaneous tissue, bone, and nail. The biquadral, plain-strain elements were used in the FE models and the commercial FE software package, ABAQUS (version 6.8), was utilized for the analyses.

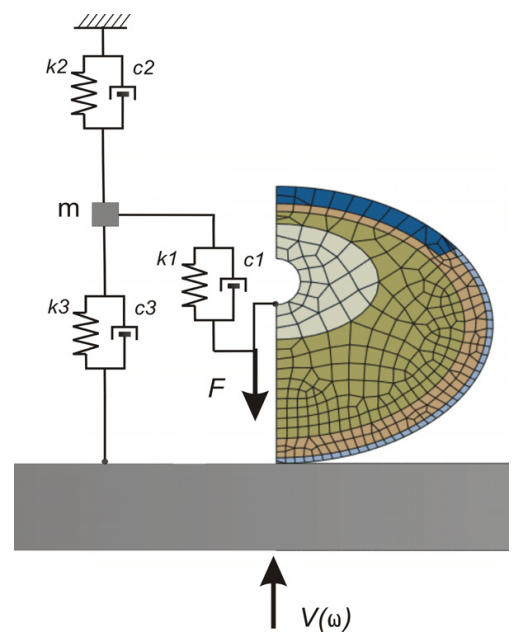

Figure 1: Hybrid model of a fingertip. The skin is assumed to be composed of two layers: the outer skin $(0.10 \mathrm{~mm}$ thick) and inner skin $(1.26 \mathrm{~mm}$ thick). The outer skin layer contains stratum corneum and a part of the viable epidermis, and is considered as linearly elastic; while the inner skin layer is composed of dermis and a part of the viable epidermis, and is characterized as nonlinearly elastic. The bone and nail are assumed to be linearly elastic [3]. Two-term MooneyRivlin models are applied to characterize the nonlinearly elastic behaviors, and the Rayleigh formula is applied for the frequency-dependent viscous damping characteristics of the soft tissues, as described in our previous studies [4,5].

The lumped element parameters were determined by fitting the model to experimental measurements. The vibration transmissibility of the fingertip was measured using a laser vibrometer (Polytec PSV-300-H). The basic measurement method and testing setup were similar to those reported in a previous study [6]. Briefly, a flat, rectangular aluminum platform $(21.6 \mathrm{x}$ $12.7 \mathrm{~cm}, 1.09 \mathrm{~kg}$ ) connected to a single-axis vibration testing system (Unholtz-Dickie, TA250S032-PB) was used to deliver the vibration. The plate was pushed on with an open hand at a given static force (from $15 \mathrm{~N}$ to $30 \mathrm{~N}$ ) during the measurement.

\section{Results}

The representative results for the frequency-dependent distributions of the vibration speed magnitude and VPAD across the central line of the fingertip model are shown in Fig. 2. At the tissue/plate contact surface, the vibration velocity is constant at $8 \mathrm{~mm} / \mathrm{s}$, while the distributions of the vibration within the soft tissue depend on the frequency. When the frequency is equal to or greater than $125 \mathrm{~Hz}$, the distribution patterns of the vibration in the soft tissue varied suddenly: 
the gradients of vibration displacement and velocity across the tissue become substantially greater. The VPAD-based frequency weighting curves are calculated and shown in Fig. 3. The curves of the ISO frequency weighting and the normalized vibration transmissibility at the nail are also shown in the figure for comparison.

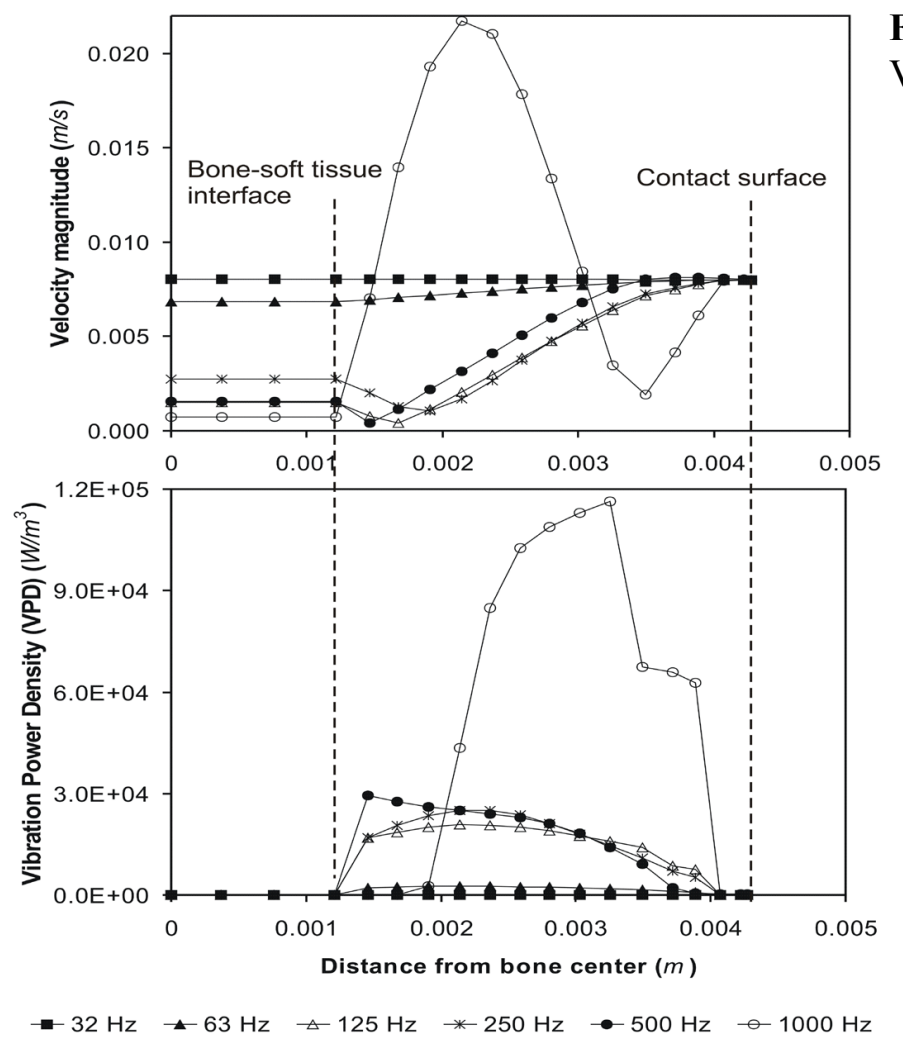

Figure 2: The distributions of vibration velocity and VPAD along the central line of the fingertip model.

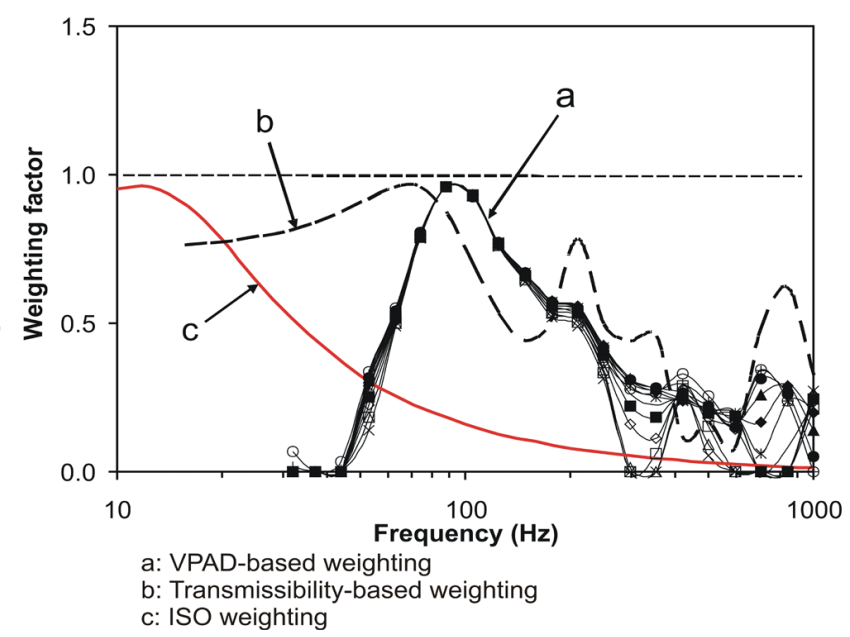

Figure 3: Comparison of the frequency weighting derived using VPAD with ISO-weighting and the weighting derived using the finger surface vibration transmissibility. The VPAD-based weightings were calculated using data at different locations within the soft tissues and shown in different symbols.

\section{Discussion and Conclusions}

As shown in Fig. 3, the VPAD frequency weighting of the finger soft tissue is largely different from the current ISO frequency weighting. If the VPAD is more closely associated with the finger disorders than the ISO weighted acceleration, the observed weighting differences suggest that the ISO weighting could overestimate the vibration effect at the low frequency but underestimate the high frequency vibration, which is consistent with the observations reported from some physiological, pathological, and epidemiological studies. It is also interesting to note that the basic trends of the finger surface vibration transmissibility at frequencies higher than 80 $\mathrm{Hz}$ are fairly consistent with the VPAD weighting, which suggest that the transmissibility could be utilized to approximately represent the weighting of the VPAD in this frequency range.

\section{Bibliography}

[1] M. Bovenzi, Vibration-induced white finger and cold response of digital arterial vessels in occupational groups with various patterns of exposure to hand-transmitted vibration, Scandinavian J.

Work, Environment \& Health 24 (2) (1998) 138-44.

[2] T. Miwa, Evaluation methods for vibration effect. Part 3: Measurement of thresholds and equal sensation contours on hand for vertical and horizontal sinusoidal vibrations, Ind Health 5 (1967) 213-20.

[3] H. Yamada, Strength of biological materials, Williams and Wilkins Co., Baltimore, 1970.

[4] J. Z. Wu, D. E. Welcome, R. G. Dong, Three-dimensional finite element simulations of the mechanical response of the fingertip to static and dynamic compressions, Comput Methods Biomech Biomed Engin 9 (1) (2006) 55-63.

[5] J. Z. Wu, R. G. Dong, D. E. Welcome, Analysis of the point mechanical impedance of fingerpad in vibration, Med Eng Phys 28 (8) (2006) 816-26.

[6] E. Concettoni, M. Griffin, The apparent mass and mechanical impedance of the hand and the transmission of vibration to the fingers, hand, and arm, J Sound Vib. 325(3) (2009) 664-678.

DISCLAIMERS: The findings and conclusions in this report are those of the authors and do not necessarily represent the views of the National Institute for Occupational Safety and Health. 